\title{
Creating an ideal "off-test mode" for rotary left ventricular assist devices: Establishing a safe and appropriate weaning protocol after myocardial recovery
}

\author{
Masahiko Ando, MD, PhD, ${ }^{\text {a,b }}$ Takashi Nishimura, MD, PhD, ${ }^{\mathrm{c}}$ Yoshiaki Takewa, MD, PhD, ${ }^{\mathrm{a}}$ \\ Shunei Kyo, MD, PhD, ${ }^{\mathrm{c}}$ Minoru Ono, MD, PhD, ${ }^{\mathrm{b}}$ Yoshiyuki Taenaka, MD, $\mathrm{PhD},{ }^{\mathrm{a}}$ and \\ Eisuke Tatsumi, MD, $\mathrm{PhD}^{\mathrm{a}}$
}

\begin{abstract}
Objective: Recent developments in adjunctive therapy have enabled us to remove once-implanted left ventricular assist devices. For successful device removal, appropriate patient selection and precise evaluation of cardiac function are essential. However, with rotary pumps, pump weaning inevitably creates diastolic retrograde pump flow, and this flow may overload the native heart. We developed a novel "off-test mode" for weaning, which increases revolutions per minute just in diastole. The present study evaluated the effect of this mode on retrograde pump flow and ventricular workload.
\end{abstract}

\begin{abstract}
Methods: The EVAHEART device (Sun Medical Technology Research Corporation, Nagano, Japan) was installed in 8 goats $(63.0 \pm 7.3 \mathrm{~kg})$. We evaluated myocardial oxygen consumption, pressure volume area, and retrograde pump flow under 3 conditions: circuit clamp, continuous mode, and off-test mode. In continuous mode, revolution per minute was set for a mean net pump flow of zero, whereas in off-test mode, systolic revolution per minute was kept at a minimum level and diastolic revolution per minute was adjusted to ensure nearzero diastolic retrograde pump flow.
\end{abstract}

Results: In off-test mode, the pressure volume loop shapes were similar to those under circuit-clamp conditions, with both myocardial oxygen consumption and pressure volume area approximately equal to those under clamp conditions. Retrograde pump flow was significantly decreased in off-test compared with continuous mode $(P=.005)$.

Conclusions: Off-test mode decreased retrograde flow during weaning while keeping ventricular workload similar to baseline. This mode is potentially valuable for establishing a safe and appropriate pump weaning protocol after myocardial recovery. Investigations on heart failure models are ongoing. (J Thorac Cardiovasc Surg 2012;143:1176-82)

Rotary left ventricular assist devices (LVADs) have greatly improved the outcomes of patients with end-stage heart failure, ${ }^{1,2}$ and recent developments in adjunctive therapy have enabled us to remove once-implanted LVADs from more patients than before. ${ }^{3-5}$ In the near future, new options, such as stem cell induction ${ }^{6}$ or gene therapy, ${ }^{7}$ should accelerate myocardial recovery and may further increase the rate of successful LVAD withdrawal.

However, to avoid removal failure, appropriate patient selection and precise evaluation of native heart function are essential. ${ }^{4,8,9}$ With pulsatile pumps, which occasionally have extracorporeal pump circuits or mechanical valves to

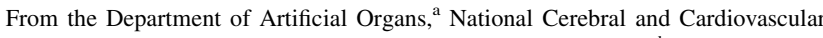
Center Research Institute, Department of Cardiothoracic Surgery, ${ }^{b}$ and Department of Therapeutic Strategy for Heart Failure, ${ }^{\mathrm{c}}$ The University of Tokyo, Japan.

Disclosures: Authors have nothing to disclose with regard to commercial support.

Received for publication April 4, 2011; revisions received June 28, 2011; accepted for publication July 26, 2011; available ahead of print Dec 5, 2011.

Address for reprints: Masahiko Ando, MD, PhD, National Cardiovascular Center Research Institute, Department of Artificial Organ, 5-7-1 Fujishiro-dai, Suita, Osaka 565-8565, Japan (E-mail: masandoo@ hotmail.com).

$0022-5223 / \$ 36.00$

Copyright $($ c 2012 by The American Association for Thoracic Surgery doi:10.1016/j.jtcvs.2011.07.065
}

prevent retrograde pump flow (PF), we can clamp the circuits or stop the driving pumps and evaluate cardiac function without support. ${ }^{10}$ The judgment should be made promptly, because no PF in the pulsatile system correlates with a high risk of thrombus formation. ${ }^{8}$ With rotary pumps, which are widely used at present, ${ }^{11}$ circuit clamping is virtually impossible, and taking down pumps inevitably creates a degree of retrograde PF in the diastolic phase, when pressure head (ie, aortic pressure [AoP] - left ventricular [LV] pressure) becomes relatively high. Among the rotary LVADs, axial-flow pumps produce minimal retrograde PF, such that they can be stopped or briefly driven at a low number of revolutions per minute (RPM), allowing a removal decision to be made. ${ }^{4,11}$ However, with centrifugal pumps, this decision can be made only by gradually decreasing RPM and carefully establishing low-flow conditions. A mean PF of zero can result in overloading of the native heart and, in the worst case, aggravate its function. ${ }^{12}$ Schima and colleagues ${ }^{10}$ reported that an assist flow of 1.0 to $1.5 \mathrm{~L} / \mathrm{min}$ requires work equivalent to that of the unsupported heart in a computer model analysis. However, this condition definitely differs among pump systems, and the optimal weaning protocol for rotary pumps has yet to be established. 

Abbreviations and Acronyms
$\mathrm{AoF}=$ aortic flow
AoP $=$ aortic pressure
$\mathrm{CVP}=$ central venous pressure
$\mathrm{ECG}=$ electrocardiography
EDP = end-diastolic pressure
$\mathrm{EDV}=$ end-diastolic volume
$\mathrm{ESV}=$ end-systolic volume
$\mathrm{LV}=$ left ventricular
LVAD = left ventricular assist device
MVO2 = myocardial oxygen consumption
$\mathrm{PF} \quad=$ pump flow
$\mathrm{PV}=$ pressure volume
PVA $=$ pressure volume area
$\mathrm{RPM}=$ revolutions per minute

Thus, we developed a novel drive mode for centrifugal pumps that can change its RPM in synchronization with heart beats. If we can increase RPM just in diastole, we may be able to control this backward flow. This novel system may help to create the ideal "off-test mode" for a safe and appropriate LVAD weaning protocol. The ideal off-test mode we are suggesting achieves both minimal support in the systolic phase and no forward or backward flow in the diastolic phase. In other words, "zero-total-flow" is created as if circuits are clamped, while keeping the circuits open and pumps driving. We previously reported the effects of this mode on retrograde PF during weaning in mock systems. ${ }^{12,13}$ In our experiments, the off-test mode effectively decreased intracircuit retrograde flow in mock circulatory systems. The present study was designed to test this novel system of centrifugal pumps in acute animal models and to evaluate the effects of this mode on retrograde PF and LV workload, or myocardial oxygen consumption (MVO2) and pressure volume area (PVA).

\section{MATERIALS AND METHODS}

\section{Experimental Preparation}

Experiments were performed on 8 goats $(63.0 \pm 7.3 \mathrm{~kg})$ sedated with an intramuscular injection of ketamine $(10 \mathrm{mg} / \mathrm{kg})$. General anesthesia was maintained by inhalation of isoflurane. The animals were fixed in the right recumbent position and mechanically ventilated. Inhaled oxygen was controlled to achieve a target arterial oxygen pressure of 100 to 150 $\mathrm{mm} \mathrm{Hg}$. The heart was approached via a left thoracotomy, and pressure lines for AoP and central venous pressure (CVP) monitoring were established from the left internal thoracic artery and vein. We placed an $18-\mathrm{mm}$ electromagnetic flow probe (EMF-1000, Nihon Kohden, Tokyo, Japan) on the ascending aorta for ascending aortic flow (AoF) monitoring and a 3-mm ultrasonic flow probe (HQD3FSB, Transonic Systems Inc, New York, NY) on the main trunk of the left coronary artery for coronary flow monitoring. A 16-mm outflow cannula was sutured to the descending aorta, and a 16-mm ultrasonic flow probe (TS420, Transonic Systems Inc) was placed around the outflow cannula for PF monitoring. After heparinization (300 U/kg), the LV apex was removed with a $19-\mathrm{mm}$ puncher, and a 20-mm inflow cannula was inserted. Both outflow and inflow cannulae were connected to the EVAHEART device (Sun Medical Technology Research Corporation, Nagano, Japan), ${ }^{14}$ and left heart bypass was started. A 4F Mikro-Tip catheter pressure transducer (Millar Instruments, Houston, Tex) and $6 \mathrm{~F}$ conductance catheter (2S-RH-6DA-116, 6F, $6 \mathrm{~mm}$, Taisho Biomed Instrument Co, Ltd, Osaka, Japan) were inserted from the anterior wall of the LV for pressure volume (PV) loop monitoring. The inferior vena cava was encircled for preload reduction to obtain an end-systolic PV relationship. A pacing lead for detection of ventricular electrocardiography (ECG) was sutured on the anterior wall of the right ventricle (Figure 1, A).

After these preparations, we waited for at least 30 minutes to ensure hemodynamic stability. All experimental protocols were approved by the Animal Research Committee of the National Cerebral and Cardiovascular Center Research Institute and conducted according to its veterinary guidelines.

\section{Experimental Protocol}

During data collection, target preload was set at $10 \mathrm{~mm} \mathrm{Hg}$ by mean CVP, and we controlled the infusion volume to maintain this level. Target heart rate and systolic pressure were 60 to 90 beats $/ \mathrm{min}$ and 80 to $120 \mathrm{~mm}$ $\mathrm{Hg}$, respectively. If these parameters deviated from their targets, we adjusted the concentration of inhaled isoflurane. No vasopressive agents were used. Lidocaine $(1 \mathrm{mg} / \mathrm{kg} / \mathrm{h})$ was continuously infused to prevent ventricular arrhythmias. We evaluated retrograde PF and LV workload under 3 conditions: 1) circuit clamp (no pump support), 2) continuous mode (constant RPM), and 3) off-test mode (increasing RPM in diastole). RPM settings for these modes are described in detail below. We waited 5 minutes to obtain hemodynamic stability after setting up the drive mode. Pressure and flow data were collected in Labchart5 (ADInstruments Pty Ltd, Bella Vista, Australia). We analyzed PF waveforms. Negative PF was integrated by time, and retrograde PF was calculated and shown as the amount per minute. At completion of the experiments, the hearts were excised, and the LV was weighed for weight normalization of MVO2 and PVA per 100 grams of LV tissue.

\section{Drive Mode}

In continuous mode, RPM was set to establish a mean PF of approximately $0 \mathrm{~L} / \mathrm{min}$. In off-test mode, we used the following novel ECGsynchronized RPM change mode. Direct ECG detection from a ventricular lead for synchronization in pulsatile LVADs was previously reported,${ }^{15}$ but direct ECG-synchronized RPM change for rotary LVADs is rare according to our search of the literature, and we are currently developing various clinical applications for this system. ${ }^{16,17}$

The details of our novel pump controller have been reported. ${ }^{13}$ Briefly, the controller can detect R waves from the ventricular ECG and instantly increase RPM to the target speed after configuration of the delay time from the $\mathrm{R}$ wave. The primary goal of our off-test mode is achieving both minimal systolic support and prevention of diastolic retrograde PF. Therefore in off-test mode, systolic RPM was set at 700, which is the minimum RPM of the current system, and diastolic RPM was set to ensure diastolic PF near $0 \mathrm{~L} / \mathrm{min}$. We adjusted this diastolic RPM manually while monitoring the PF curve. The time that the target speed is maintained can also be configured. We defined the systolic phase as $33 \%$ of the RR interval and the diastolic phase as the remaining $67 \%$. Thus, in off-test mode, delay time was set as the systolic phase, or $33 \%$ of the RR interval. For example, at a native heart rate of 90 beats/min, the RR interval is $670 \mathrm{msec}$, with $33 \%$ being $220 \mathrm{msec}$. Pumps were thus driven at a systolic RPM of 700 from the R-wave input for $220 \mathrm{msec}$, and diastolic RPM was instantly increased to achieve a diastolic PF of approximately $0 \mathrm{~L} / \mathrm{min}$. We maintained this diastolic RPM for the other $67 \%$ of the RR interval, or 450 $\mathrm{msec}$, and finally returned to the initial systolic RPM of $700 \mathrm{rpm}$. We calculated the bypass rate by dividing PF by the sum of PF and AoF, as shown in "Results." 


\section{$\leftarrow$ Cranial Caudal $\rightarrow$}

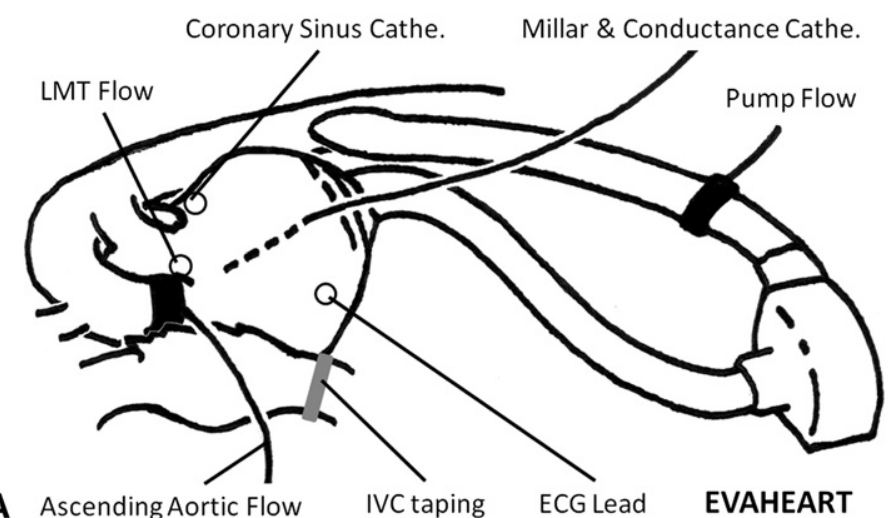

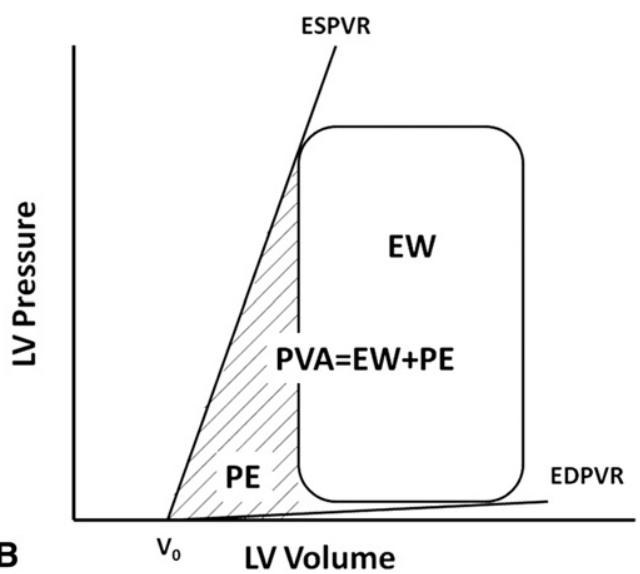

B

\section{Volume}

FIGURE 1. A, Experimental settings. B, PV loop. PVA is the sum of external work and potential energy. LMT, Left main trunk (of the coronary artery); $I V C$, inferior vena cava; $E C G$, electrocardiogram; $E S P V R$, end-systolic pressure volume relationship; $L V$, left ventricular; $E W$, external work; $P E$, potential energy; $P V A$, pressure volume area; $E D P V R$, end-diastolic pressure volume relationship; $V_{0}, \mathrm{X}$ axis intercept of the end-systolic $\mathrm{PV}$ relationship slope.

\section{Myocardial Oxygen Consumption Assessment}

We calculated MVO2 for evaluation of LV workload. ${ }^{16,18-20}$ A 10F retrograde cardioplegic catheter (Gandry RCSP catheter, 94110, Medtronic Japan Co, Ltd, Tokyo, Japan) was inserted into the coronary sinus through the hemiazygos vein. A balloon was inflated, and coronary vein blood was drawn via a small hole created manually just before a balloon to avoid collecting mixed central venous blood. ${ }^{18}$ We calculated MVO2 by the following formula: MVO2 $(\mathrm{O} 2 \mathrm{~mL} / \mathrm{min})=\{(\mathrm{ScaO} 2-$ $\mathrm{ScvO} 2) \cdot 1.34 \cdot \mathrm{aHb}+(\mathrm{PcaO} 2-\mathrm{PcvO} 2) \cdot 0.003\} \cdot 0.01 \cdot$ coronary flow, where $\mathrm{ScaO} 2(\mathrm{ScvO} 2)$ is the oxygen saturation of coronary artery (vein) blood, $\mathrm{PcaO} 2$ ( $\mathrm{PcvO} 2), \mathrm{mm} \mathrm{Hg}$ is the partial pressure of coronary artery (vein) blood, and $\mathrm{aHb}, \mathrm{g} / \mathrm{dL}$ is the hemoglobin concentration of coronary artery blood.

We calculated normalized MVO2 per beat per $100 \mathrm{~g}$ of LV tissue. Data are presented as percentages of those in the circuit clamp, taken as $100 \%$.

\section{Pressure Volume Area Assessment}

The Sigma5 DF system (CD Leycom, Zoetermeer, The Netherlands) was used for PV loop analysis. The end-systolic PV relationship slope was obtained by gradually occluding the inferior vena cava. PVA, recognized as a measure of total mechanical energy, ${ }^{19,20}$ was approximated using a previously established method. ${ }^{20,21}$ Briefly, external work is a computed area in the loop and potential energy is a crescent-shaped area between the end-systolic PV relationship slope and isovolumic relaxation (Figure 1, B). PVA, the sum of external work and potential energy, is shown as the normalized value per $100 \mathrm{~g}$ of $\mathrm{LV}$ tissue. ${ }^{20} \mathrm{We}$ also evaluated end-systolic pressure, end-systolic volume (ESV), end-diastolic pressure (EDP), and end-diastolic volume (EDV). We drew blood and performed calibration with a conductivity meter. However, the absolute LV volume value does not reflect actual LV volume. Therefore, we evaluated these data by comparative assessment with LV volume under circuit-clamp conditions as the baseline $(100 \%)$. LV pressure data are also presented as percent values.

\section{Statistical Analysis}

All numeric data are shown as average \pm standard deviation. Comparisons between groups were performed by repeated-measures analysis of variance followed by Tukey's multiple comparison test. All analyses were conducted using a 2-sided method. The efficacy outcome measure was the retrograde $\mathrm{PF}$ amount. We specified a minimum detectable difference in retrograde $\mathrm{PF}$ of $0.75 \mathrm{~L} / \mathrm{min}$ (the difference between a mean of 1.40 $\mathrm{L} / \mathrm{min}$ for continuous mode and $0.65 \mathrm{~L} / \mathrm{min}$ for off-test mode). Therefore, a sample size of 8 was selected to ensure $80 \%$ power for detecting the specified minimum difference at a 2-sided significance level of .05. We used PASW Statistics18 (IBM SPSS Inc, Chicago, Ill) for all statistical analyses.

\section{RESULTS}

Figure 2 shows sample waveforms of pressure and flow data. In continuous mode, diastolic reverse PF was observed and diastolic AoP shifted upward compared with the circuit clamp (Figure 2). While in off-test mode, diastolic RPM was increased by synchronization and the reversal of PF almost completely disappeared. Diastolic AoP was maintained in off-test mode.

Table 1 shows numeric flow and pressure data. There was no significant change in heart rate, mean CVP, mean AoP, or mean LV pressure. In continuous mode, RPM was approximately 990 and PF was $0.19 \mathrm{~L} / \mathrm{min}$ (Table 1). In off-test mode, systolic RPM was 690 and diastolic RPM was 1320. $\mathrm{PF}$ increased to 0.66 from $0.19 \mathrm{~L} / \mathrm{min}$ in continuous mode, but the difference was not significant. In off-test mode, AoF was significantly decreased and the bypass rate was significantly increased compared with continuous mode (Table 1).

PV loops are shown in Figure 3. In continuous mode, both end-systolic and diastolic points were shifted rightward compared with the circuit-clamp. End-diastolic points also shifted upward. While in off-test mode, the loop was similar to that under circuit-clamp conditions. Ventricular pressures and volumes are shown in Figure 4. In continuous mode, $\%$ EDP was increased $(P=.001) . \%$ EDP decreased in off-test mode, but the difference was not significant $(P=.08)$. Both \%EDV and \% ESV increased in continuous mode, but these differences also did not reach statistical significance.

$\% \mathrm{PVA}$ and \%MVO2 in each mode are shown in Figure 5, A. \% PVA increased in continuous mode, but the difference was not significant. In off-test mode, \%PVA 


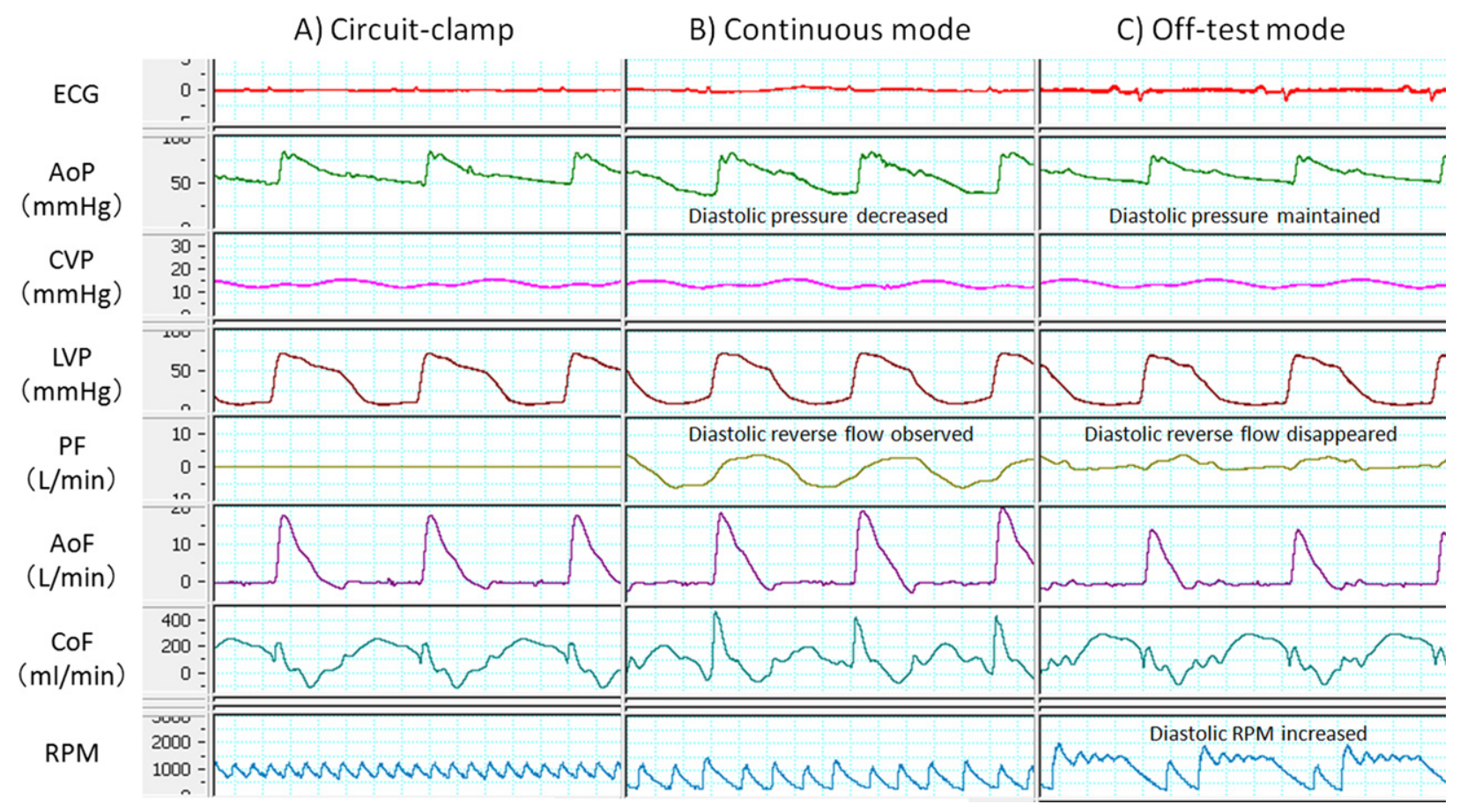

FIGURE 2. Pressure and flow waveforms. In continuous mode, diastolic reverse PF was observed. However in off-test mode, diastolic RPM increased and the reversal of $\mathrm{PF}$ disappeared completely. $E C G$, Electrocardiogram; $A o P$, aortic pressure; $C V P$, central venous pressure; $L V P$, left ventricular pressure; $P F$, pump flow; $A o F$, ascending aortic flow; $C o F$, coronary flow; $R P M$, revolution per minute.

and $\% \mathrm{MVO} 2$ were both similar to corresponding values under circuit-clamp conditions. The amount of retrograde PF in each mode is shown in Figure 5, B. In continuous mode, the amount was $1.38 \pm 0.62 \mathrm{~L} / \mathrm{min}$, and significantly decreased to $0.66 \pm 0.57 \mathrm{~L} / \mathrm{min}(P=.005)$ in off-test mode.

\section{DISCUSSION}

Our ultimate goal is to establish a safe and appropriate weaning protocol for rotary LVAD removal. Our novel drive mode that can change pump RPM via synchronization successfully reduced the amount of retrograde PF in diastole during pump weaning while keeping LV workload similar to that under circuit-clamp conditions. These data raise

TABLE 1. Flow and pressure data

\begin{tabular}{lccl}
\hline \multicolumn{1}{c}{ Mode } & $\begin{array}{c}\text { Circuit } \\
\text { clamp }\end{array}$ & $\begin{array}{c}\text { Continuous } \\
\text { mode }\end{array}$ & Off-test mode \\
\hline Heart rate $(\mathrm{rpm})$ & $78.0 \pm 13.8$ & $77.9 \pm 13.5$ & $75.6 \pm 13.9$ \\
Mean CVP $(\mathrm{mm} \mathrm{Hg})$ & $11.8 \pm 5.3$ & $11.7 \pm 4.9$ & $12.3 \pm 4.6$ \\
Mean AoP (mm Hg) & $62.4 \pm 11.2$ & $56.3 \pm 8.8$ & $57.1 \pm 12.4$ \\
Mean LVP (mm Hg) & $38.9 \pm 11.7$ & $38.9 \pm 11.4$ & $37.5 \pm 10.7$ \\
Systolic RPM & $0.0 \pm 0.0$ & $990 \pm 210^{*}$ & $690 \pm 60^{*}, \dagger$ \\
Diastolic RPM & $0.0 \pm 0.0$ & $990 \pm 210^{*}$ & $1320 \pm 290^{*}, \dagger$ \\
Mean RPM & $0.0 \pm 0.0$ & $960 \pm 190^{*}$ & $1060 \pm 210^{*}$ \\
PF (L/min) & $0.00 \pm 0.00$ & $0.19 \pm 0.37$ & $0.66 \pm 0.85$ \\
AoF (L/min) & $3.94 \pm 1.93$ & $3.68 \pm 1.89$ & $2.75 \pm 1.32^{*}, \dagger$ \\
Bypass rate $(\%)$ & $0.0 \pm 0.0$ & $5.7 \pm 10.6$ & $19.5 \pm 14.5^{*}, \dagger$ \\
\hline
\end{tabular}

$L V P$, Left ventricular pressure. *Significant change from circuit-clamp condition. $\dagger$ Significant change from continuous mode. the possibility of mimicking circuit-clamp conditions while evaluating native heart function before LVAD removal, even in rotary LVADs implanted intracorporeally. These findings are both unique and clinically relevant.

In addition to the weaning protocol, other factors, such as patient selection and effective adjunctive therapies, are clearly of great significance. Dandel and colleagues ${ }^{4}$ reported pre-explantation factors, including duration of LVAD support, heart failure duration before LVAD implantation, and echocardiographic findings, to predict cardiac stability after LVAD removal. Birks and colleagues 3,9 have suggested the importance of medical therapy, including the beta- 2 agonist clenbuterol in combination with mechanical support. ${ }^{3,9}$ Other medical therapies, ${ }^{22}$ resynchronization therapy, ${ }^{23}$ or rehabilitation ${ }^{23}$ during VAD support augment the rate of successful removal. In the near future, even molecular changes occurring during reverse remodeling might be of value in assessing device withdrawal. ${ }^{24}$ Nevertheless, creating or mimicking nosupport circumstances before removal would likely still be necessary.

Figure 2 shows sample pressure and waveforms. In continuous mode, retrograde PF persisted and diastolic AoP was shifted downward, possibly because of this retrograde PF. In off-test mode, the amount of retrograde PF decreased as diastolic RPM increased, and diastolic AoP was similar to that under circuit-clamp condition. Comparing PF wave forms between continuous and off-test modes showed 
Circuit-clamp

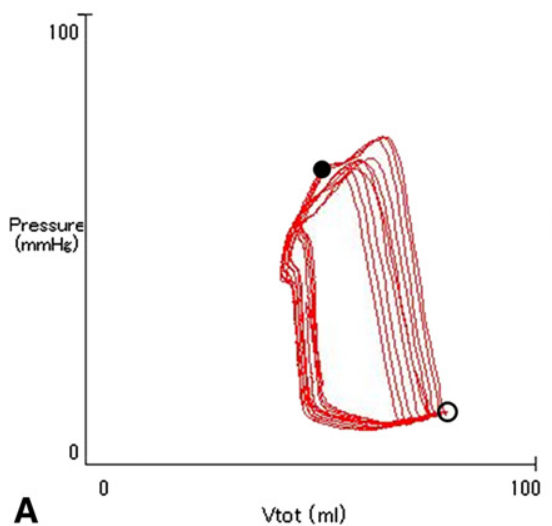

Continuous mode

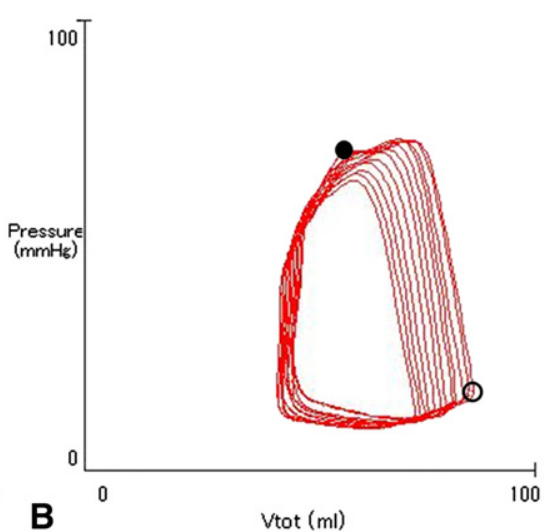

Off-test mode

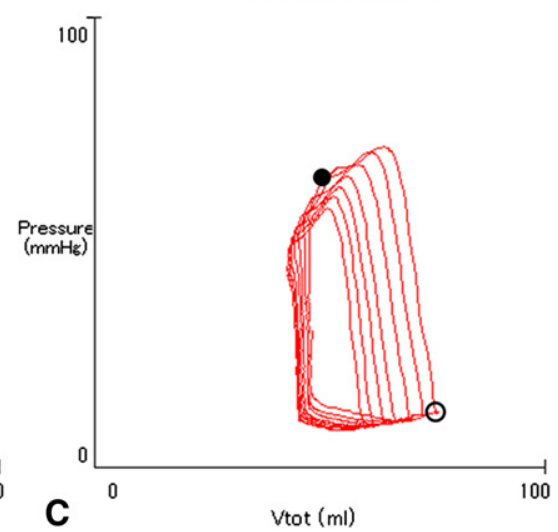

FIGURE 3. PV loops. End-systolic point (black dot) and end-diastolic point (white circle). In continuous mode, end-systolic and end-diastolic points are shifted rightward compared with circuit-clamp conditions. End-diastolic points are shifted upward. In off-test mode, the loop is similar to that in circuitclamp condition.

almost zero PF, whereas continuous mode showed "zero net flow," meaning certain amounts of both forward and backward flow persisted. While in off-test mode, an almost flat PF waveform was obtained, and this mode showed "zerototal-flow in the circuit," as if the circuit was clamped. This was the PF waveform we were endeavoring to achieve in the current study (Figure 2).
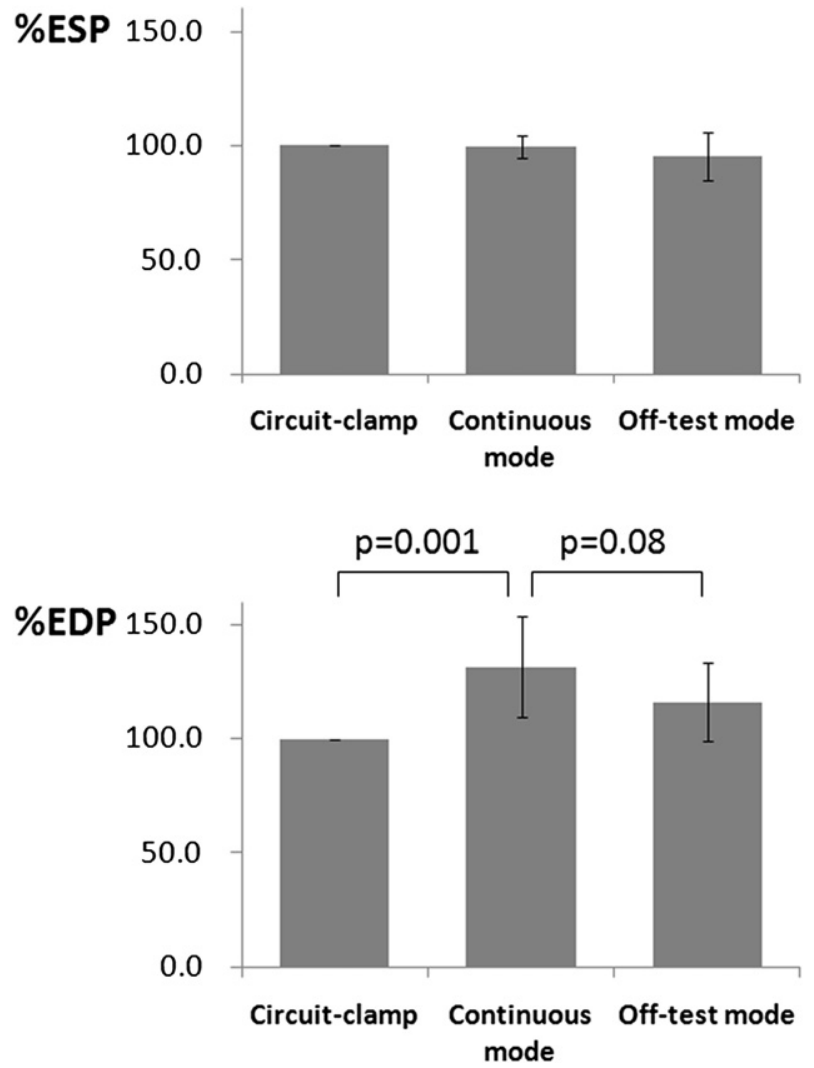

The coronary flow waveform in off-test mode was similar to that under circuit-clamp conditions, whereas that in continuous mode was entirely different from those in the other 2 states, in that diastolic coronary flow was decreased (Figure 2). We speculate that in continuous mode, LVED pressure was increased because of retrograde PF (Figure 4), and diastolic coronary flow could
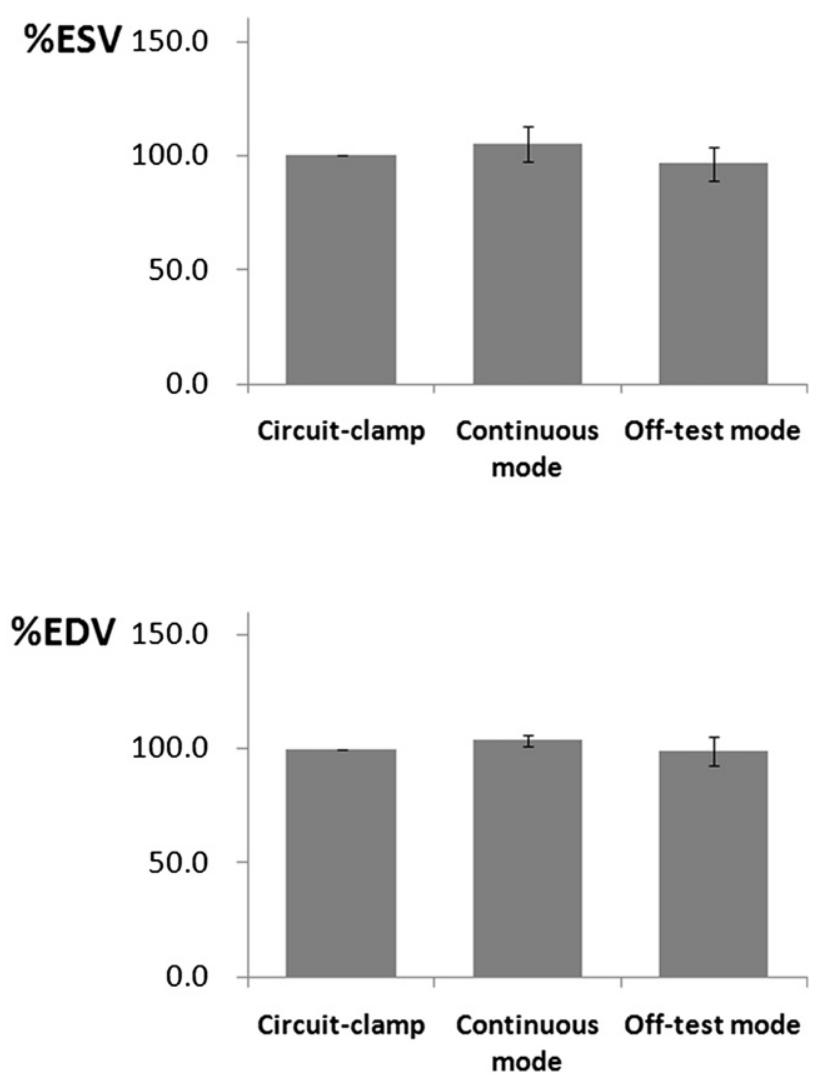

FIGURE 4. LV pressure and volume data. These parameters are shown as percentages of values under circuit-clamp conditions as baseline (100\%). \%EDP was significantly increased in continuous mode compared with circuit-clamp conditions. $E S P$, End-systolic pressure; $E S V$, end-systolic volume; $E D P$, enddiastolic pressure; $E D V$, end-diastolic volume. 

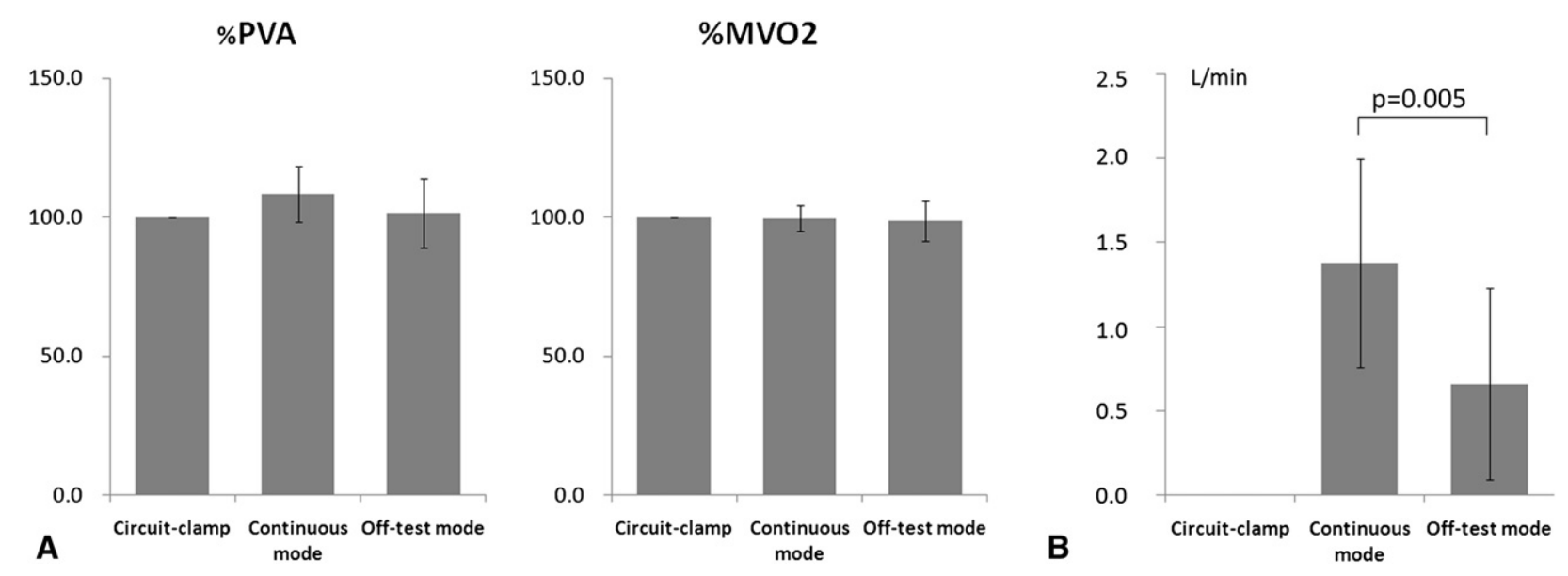

FIGURE 5. A, PVA and MVO2. There were no significant changes in PVA or MVO2 in any of the 3 modes. B, Retrograde PF. In continuous mode, the amount of reverse PF was $1.38 \mathrm{~L} / \mathrm{min}$. However, this was significantly decreased to $0.66 \mathrm{~L} / \mathrm{min}$ in off-test mode. PVA, Pressure volume area; $M V O 2$, myocardial oxygen consumption.

have been decreased by increased intramural pressure. $^{19,25}$

Table 1 shows flow and pressure numeric data. There were no changes in heart rate, CVP, AoP, or LV pressure. In off-test mode, PF was increased compared with continuous mode. This change, attributed to reduced retrograde $\mathrm{PF}$, was not significant. In off-test mode, AoF was significantly decreased (Table 1). This AoF decrease may have also been due to reduced retrograde PF. The present study used a normal heart model, such that in continuous mode the native heart could compensate for backward flow by increasing AoF. This compensation could represent an excessive load on the native heart during pump weaning. While in offtest mode, there was almost no reverse flow, making compensation by the native heart unnecessary. In the systolic phase, it would be natural for a small amount of forward flow to occur in off-test mode (Figure 2), because the pump circuit is open. We believe this systolic forward flow was ejected mostly by the native heart because systolic RPM in off-test mode was set at 700, the minimum RPM in the current system (Table 1).

Figure 3 shows sample PV loops, and Figure 5, A, shows changes in \% PVA and \%MVO2. We were successful in obtaining similar PV loops in off-test mode and circuit-clamp states, meaning that the native heart workload was almost the same in these 2 settings (Figure 5, A). In continuous mode, the PV loop was larger than under the other 2 conditions, but this change was not significant. We speculate that the lack of significant change in LV workload is attributable to good ventricular function. The results would have been different had we conducted the same investigation in a heart failure model. Figure 4 shows LV pressure and volume. In continuous mode, EDP was significantly increased compared with circuit-clamp conditions $(P=.001)$, and offtest mode blunted this elevation in $\operatorname{EDP}(P=.08)$. EDV and ESV were unchanged, possibly because of low compliance of the native heart and the rate of LV volume change. In continuous mode, EDP occasionally doubled, for example, from 10 to $20 \mathrm{~mm} \mathrm{Hg}$, but there was no increase in EDV or ESV even with such a large change. Figure 5, $B$, shows changes in retrograde PF, indicating off-test mode to successfully reduce retrograde PF. The retrograde PF difference between the 2 modes was modest, but this difference might be larger in heart failure models, possibly affecting native heart recovery.

There have been clinical reports describing pump-off trials after myocardial recovery., ${ }^{3,4,11}$ In patients with pulsatile pumps, Mano and colleagues ${ }^{8}$ conducted dobutamine stress testing under minimal support with pulsatile LVADs, driven at 60 beats $/ \mathrm{min}$. Dandel and colleagues ${ }^{4}$ performed 10-minute echocardiography under support at 1 beat/min, which necessitated systemic heparinization. With most pulsatile LVADs, there is little concern about retrograde PF because of the mechanical valves in circuits, and with commonly used pulsatile pumps, such as the Heartmate XVE (Thoratec Corp, Pleasanton, Calif), pump-off trials can be attempted using intermittent hand pumping with systemic anticoagulation. However, these trials should be done relatively quickly to avoid intracircuit clotting. With rotary LVADs, attempts have been made to adjust pump RPM to achieve "zero net flow" condition, tolerating both forward and backward flows in the circuit. ${ }^{4}$ However, this approach to pump weaning raises concerns, because retrograde $\mathrm{PF}$ in diastole might overload the native heart and even be misleading for cardiac evaluation. ${ }^{10}$ As shown in the current study, continuous mode with low RPM actually created a certain amount of retrograde PF (Figure 2), and our off-test mode blunted this retrograde PF.

Even in the current era, weaning tests should be done on admission under careful monitoring with a pulmonary artery catheter and echocardiography, relatively quickly because of the aforementioned retrograde PF during 
weaning. However, our off-test mode decreased this reverse flow in a normal heart model. Before the clinical application of this system, we must clarify its utility in heart failure models. In the near future, it should be possible to more safely maintain this mode for a relatively long time, even a few months. Our ultimate goal is establish a safe and appropriate weaning protocol for LVAD withdrawal that can be carried out in the outpatient setting. The aims of an off-test trial in the outpatient clinic would be (1) to allow more time to safely decide on withdrawal, with careful patient selection; (2) to take into account patients' activities of daily living and symptoms in their own daily lives before pump removal; and (3) to develop a reversible mode allowing a return to full support conditions in the event of worsening patient status.

\section{Study Limitations}

The present study has several limitations. First, we need to establish the method of actual adjustment of diastolic RPM in the clinical setting. With the current system, PF monitoring during pump support is difficult. In practice, we were able to measure the amount of retrograde PF by echocardiography in the outpatient clinic, allowing diastolic RPM to be adjusted. Second, our mode might result in stagnant flow within the circuit. Therefore, we must provide adequate anticoagulation with Coumadin (Bristol-Myers Squibb, Princeton, NJ) while using the off-test mode. Otherwise, intracircuit stagnant flow might be washed out by intermittently increasing RPM. A study for evaluating chronic thrombus formation is currently ongoing. Third, the study was conducted using a normal heart model and a relatively small sample number of animals. We will next focus on a heart failure model, in which the native heart cannot compensate for retrograde PF in continuous mode, such that PVA and MVO2 will increase more than in the present study. Further investigations will examine chronic heart failure models and include larger sample sizes, providing more information on clotting or device durability.

\section{CONCLUSIONS}

Our newly developed off-test mode for centrifugal LVADs can decrease retrograde PF during pump weaning while keeping LV workload similar to that under circuitclamp conditions. This mode is of potential value in establishing a safe and appropriate LVAD weaning protocol after myocardial recovery. Further investigation in chronic heart failure models is currently ongoing.

\section{References}

1. Slaughter MS, Rogers JG, Milano CA, Russell SD, Conte JV, Feldman D, et al. Advanced heart failure treated with continuous-flow left ventricular assist device. N Engl J Med. 2009;361:2241-51.

2. Lahpor J, Khaghani A, Hetzer R, Pavie A, Friedrich I, Sander K, et al. European results with a continuous-flow ventricular assist device for advanced heart-failure patients. Eur J Cardiothorac Surg. 2010;37:357-61.
3. Birks EJ, Tansley PD, Hardy J, George RS, Bowles CT, Burke M, et al. Left ventricular assist device and drug therapy for the reversal of heart failure. $N$ Engl J Med. 2006;355:1873-84.

4. Dandel M, Weng Y, Siniawski H, Potapov E, Drews T, Lehmkuhl HB, et al. Prediction of cardiac stability after weaning from left ventricular assist devices in patients with idiopathic dilated cardiomyopathy. Circulation. 2008;118: S94-105.

5. Matsumiya G, Monta O, Fukushima N, Sawa Y, Funatsu T, Toda K, et al. Who would be a candidate for bridge to recovery during prolonged mechanical left ventricular support in idiopathic dilated cardiomyopathy? J Thorac Cardiovasc Surg. 2005;130:699-704.

6. Mokashi SA, Guan J, Wang D, Tchantchaleishvili V, Brigham M, Lipsitz S, et al. Preventing cardiac remodeling: the combination of cell-based therapy and cardiac support therapy preserves left ventricular function in rodent model of myocardial ischemia. J Thorac Cardiovasc Surg. 2010;140:1374-80.

7. Korf-Klingebiel M, Kempf T, Schluter KD, Willenbockel C, Brod T, Heineke J, et al. Conditional transgenic expression of fibroblast growth factor 9 in the adult mouse heart reduces heart failure mortality after myocardial infarction. Circulation. 2011;123:504-14.

8. Mano A, Nakatani T, Oda N, Kato T, Niwaya K, Tagusari O, et al. Which factors predict the recovery of natural heart function after insertion of a left ventricular assist system? J Heart Lung Transplant. 2008;27:869-74.

9. Birks EJ, George RS, Hedger M, Bahrami T, Wilton P, Bowles CT, et al. Reversal of severe heart failure with a continuous-flow left ventricular assist device and pharmacological therapy: a prospective study. Circulation. 2011;123:381-90.

10. Schima H, Vollkron M, Boehm H, Rothy W, Haisjackl M, Wieselthaler G, et al. Weaning of rotary blood pump recipients after myocardial recovery: a computer study of changes in cardiac energetics. J Thorac Cardiovasc Surg. 2004;127:1743-50.

11. Myers TJ, Frazier OH, Mesina HS, Radovancevic B, Gregoric ID. Hemodynamics and patient safety during pump-off studies of an axial-flow left ventricular assist device. J Heart Lung Transplant. 2006;25:379-83.

12. Ando M, Nishimura T, Takewa Y, Ogawa D, Yamazaki K, Kashiwa K, et al. What is the ideal off-test trial for continuous-flow ventricular-assist-device explantation? Intracircuit back-flow analysis in a mock circulation model. J Artif Organs. 2011;14:70-3. Epub 2011 Jan 18.

13. Ando M, Nishimura T, Takewa Y, Ogawa D, Yamazaki K, Kashiwa K, et al. A novel counterpulse drive mode of continuous-flow left ventricular assist devices can minimize intracircuit backward flow during pump weaning. J Artif Organs. 2011;14:74-9. Epub 2011 Jan 18.

14. Yamazaki K, Saito S, Kihara S, Tagusari O, Kurosawa H. Completely pulsatile high flow circulatory support with a constant-speed centrifugal blood pump: mechanisms and early clinical observations. Gen Thorac Cardiovasc Surg. 2007;55:158-62.

15. Kitamura M, Hanzawa K, Aoki K, Saitoh M, Hayashi J. Direct cardiac potential trigger for chronic control of a ventricular assist device. ASAIO J. 2001;47:302-4.

16. Ando M, Nishimura T, Takewa Y, Yamazaki K, Kyo S, Ono M, et al. Electrocardiogram-synchronized rotational speed change mode in rotary pumps could improve pulsatility. Artif Organs. 2011;35:941-7.

17. Ando M, Takewa Y, Nishimura T, Yamazaki K, Kyo S, Ono M, et al. A novel counterpulsation mode of rotary left ventricular assist devices can enhance myocardial perfusion. J Artif Organs. 2011;14:185-91.

18. Tuzun E, Eya K, Chee HK, Conger JL, Bruno NK, Frazier OH, et al. Myocardial hemodynamics, physiology, and perfusion with an axial flow left ventricular assist device in the calf. ASAIO J. 2004;50:47-53.

19. Tune JD, Gorman MW, Feigl EO. Matching coronary blood flow to myocardial oxygen consumption. J Appl Physiol. 2004;97:404-15.

20. Suga H, Hayashi T, Shirahata M. Ventricular systolic pressure-volume area as predictor of cardiac oxygen consumption. Am J Physiol. 1981;240:H39-44.

21. Ueno A, Tomizawa Y. Cardiac rehabilitation and artificial heart devices. J Artif Organs. 2009;12:90-7.

22. Nishimura T, Kyo S. High-dose carvedilol therapy for mechanical circulatory assisted patients. J Artif Organs. 2010;13:88-91.

23. Nishimura T, Kyo S. Triple-site pacing: a new supported therapy approach for bridge to recovery with a left ventricular assist system in a patient with idiopathic dilated cardiomyopathy. J Artif Organs. 2010;13:54-7.

24. Birks EJ, George RS. Molecular changes occurring during reverse remodelling following left ventricular assist device support. J Cardiovasc Transl Res. 2010; 3:635-42.

25. Elhabyan AK, Reyes BJ, Hallak O, Broce M, Rosencrance JG, Lucas BD, et al. Subendocardial ischemia without coronary artery disease: is elevated left ventricular end diastolic pressure the culprit? Curr Med Res Opin. 2004;20:773-7. 